\title{
Variations
}

Variations

Revue internationale de théorie critique

$23 \mid 2020$

Pour une gauche érotique

\section{L'amour dans la société nouvelle}

\section{Alexandra Kollontai}

\section{OpenEdition}

Journals

Édition électronique

URL : http://journals.openedition.org/variations/1473

DOI : 10.4000/variations. 1473

ISSN : 1968-3960

Éditeur

Les amis de Variations

Référence électronique

Alexandra Kollontai, «L'amour dans la société nouvelle », Variations [En ligne], 23 | 2020, mis en ligne le 01 septembre 2020, consulté le 07 septembre 2020. URL : http://journals.openedition.org/variations/ 1473 ; DOI : https://doi.org/10.4000/variations. 1473

Ce document a été généré automatiquement le 7 septembre 2020

Les ami•e•s de Variations 


\title{
L'amour dans la société nouvelle
}

\author{
Alexandra Kollontai
}

\section{NOTE DE L'ÉDITEUR}

Le texte d'Alexandra Kollontai fut initialement publié dans le N.47 du Bulletin communiste, novembre 1923 (partie III) aussi disponible en accès libre sur https://www.marxists.org/francais/kollontai/works/1923/05/eros.htm

1 La nouvelle société communiste laborieuse s'édifie sur le principe de camaraderie, de solidarité. Mais qu'est-ce que la solidarité ? Ce n'est pas seulement la conscience de la communauté d'intérêts, mais c'est aussi les liens de cœur et d'esprit établis entre les membres de la collectivité laborieuse. Le régime social bâti sur la solidarité et la collaboration exige cependant que la société en question possède à un très haut degré de développement "la capacité potentielle de l'amour», c'est-à-dire la capacité des sensations sympathiques.

2 A défaut de telles sensations, la solidarité ne peut être assurée. C'est pourquoi justement l'idéologie prolétarienne cherche à éduquer et à renforcer chez chaque membre de la classe ouvrière le sentiment de sympathie à l'égard des souffrances et des besoins de ses camarades de classe, ainsi que la compréhension des aspirations d'autrui et la conscience de sa liaison avec d'autres membres de la collectivité. Mais toutes ces «sensations sympathiques» - délicatesse, sensibilité, sympathie - découlent d'une même source commune : la capacité d'aimer, d'aimer non plus dans le sens étroitement sexuel, mais dans le sens plus large de ce mot.

3 L'amour est un sentiment qui lie les individus entre eux : il est donc pour ainsi dire un sentiment d'ordre organique. Que l'amour soit une très grande force de liaison, la bourgeoisie le comprenait et le saisissait très bien. C'est pourquoi, en cherchant à consolider la famille, l'idéologie bourgeoise fit une vertu morale de l' «amour entre époux » : être un "bon père de famille » était, aux yeux de la bourgeoisie, une très grande et très précieuse qualité de l'homme. 
4 Le prolétariat, de son côté, doit escompter le rôle social et psychologique que le sentiment d'amour, aussi bien dans le sens étendu du mot qu'en ce qui concerne les rapports entre les sexes, peut et doit jouer pour renforcer des liens, non dans le domaine des relations matrimoniales et de famille, mais dans celui du développement de la solidarité collective.

Quel est donc l'idéal d'amour de la classe ouvrière? Quels sont les sentiments que l'idéologie prolétarienne met à la base des rapports entre les sexes?

Nous avons déjà constaté, mon jeune ami, que chaque époque possède son propre idéal d'amour, que chaque classe cherche, dans son propre intérêt, à mettre dans la notion morale de l'amour son contenu propre. Chaque degré de civilisation apportant à l'humanité des sensations morales et intellectuelles plus richement nuancées, fait teindre en une couleur particulière les tendres ailes de l'Eros. L'évolution dans le développement de l'économie et des mœurs sociales était accompagnée des modifications qu'on apportait à la notion de l'amour. Certaines nuances de ce sentiment se renforçaient, d'autres, par contre, s'atténuaient ou disparaissaient complètement.

7 De simple instinct biologique - l'instinct de la reproduction - propre à tous les êtres vivants supérieurs ou inférieurs divisés en sexes, l'amour, évoluant depuis les milliers d'années qu'existe la société humaine, s'enrichissant sans cesse de nouvelles sensations psychiques, devint un sentiment très compliqué ${ }^{3}$. De phénomène biologique, l'amour devint un facteur social et psychologique. Sous la pression des forces économiques et sociales, l'instinct biologique de la reproduction qui détermina les rapports des sexes aux premiers degrés du développement de l'humanité s'est dirigé dans deux sens diamétralement opposés. D'une part, le sain instinct sexuel - l'attraction physique l'un vers l'autre de deux êtres de sexe différent dans le but de la reproduction - sous la pression de monstrueux rapports économiques et sociaux, surtout sous la domination du capitalisme, dégénéra en une luxure malsaine. L'acte sexuel devint un but en soi, un moyen de se procurer « une volupté de plus ", une paillardise exacerbée par les excès, les perversions, les aiguillons nocifs de la chair. L'homme s'unit à la femme non pas parce qu'un sain courant sexuel l'attire puissamment vers cette femme-là, mais parce qu'il cherche la femme, sans éprouver aucun besoin sexuel, et il la cherche dans le seul but de provoquer ce besoin, grâce à l'intimité de cette femme. Il se procure ainsi une volupté par le fait même de l'acte sexuel. La prostitution repose là-dessus. Si l'intimité de la femme ne provoque pas l'excitation attendue, les hommes, blasés par les excès sexuels, recourent à toutes sortes de perversions.

8 C'est là une déviation de l'instinct biologique vers une luxure malsaine, qui l'éloigne de sa source première.

9 D'autre part, l'attraction physique des deux sexes s'est compliquée, au courant des siècles de vie sociale de l'humanité et des changements de civilisation, de toute une gamme de sentiments. Dans sa forme actuelle, l'amour est un état psychique très complexe, depuis très longtemps déjà détaché de sa source première, l'instinct biologique de reproduction, et qui souvent se trouve même en contradiction avec lui. L'amour est un conglomérat de toute sorte de sentiments : amitié, passion, tendresse spirituelle, pitié, inclination, habitude, etc., etc. Il devient difficile, étant donnée une telle complexité, d'établir le lien direct entre «l'Eros sans ailes » (attraction physique des sexes) et «l'Éros aux ailes déployées » (attraction psychique). L'amour-amitié dans lequel il n'existe même pas un atome d'attraction physique, l'amour spirituel envers la 
cause, l'idée, l'amour impersonnel de la collectivité - tout cela témoigne que le sentiment d'amour s'est détaché de sa base biologique et à quel point il s'est idéalisé.

Mais ce n'est pas tout. Souvent, entre les diverses manifestations de l'amour, surgit une contradiction flagrante, la lutte commence. L'amour envers « la cause aimée » (non pas envers la cause tout simplement, mais justement envers la cause aimée) ne cadre pas avec l'amour envers l'élu ou l'élue du cœur) ${ }^{4}$; l'amour envers la collectivité entre en conflit avec le sentiment d'amour envers la femme, le mari, les enfants. L'amour-amitié est en contradiction avec l'amour-passion, Dans un cas, l'amour est dominé par l'harmonie psychique ; dans l'autre, il a pour base «l'harmonie des corps».

11 L'amour a revêtu de multiples aspects. Au point de vue des émotions d'amour, ce que ressent l'homme d'aujourd'hui chez lequel des siècles d'évolution culturelle ont développé et éduqué les différentes nuances de ce sentiment, se trouve à l'étroit dans ce qu'exprime le mot, trop général et vague, d'amour ${ }^{5}$.

12 La multiplicité du sentiment d'amour crée, sous la domination de l'idéologie et des mœurs bourgeoises capitalistes, une série de pénibles et insolubles drames moraux. Déjà à partir de la fin du XIX ${ }^{e}$ siècle, la multiplicité du sentiment d'amour devint le thème favori des écrivains psychologues. "L'amour envers deux » et même "envers trois ", préoccupait et embarrassait par son "énigme » les nombreux représentants réfléchis de la culture bourgeoise. Cette complexité de l'âme, ce dédoublement du sentiment, notre grand penseur publiciste A. Herzen, a cherché à les élucider dans les années du siècle passé, dans son roman intitulé: A qui la faute? Tchernychevski, également, a cherché a résoudre ce problème, dans sa nouvelle sociale : Que faire? . Le dédoublement du sentiment d'amour, sa multiplicité ont souvent préoccupé les plus grands écrivains de la Scandinavie : Hamsun, Ibsen, Bjørnson ${ }^{6}$, Geijerstam. Ce thème, on le rencontre souvent dans les écrits des littérateurs français du siècle dernier. Romain Rolland, très proche du communisme, s'en occupe aussi bien que Maeterlinck, très éloigné de nos conceptions ${ }^{7}$.

13 Les génies poétiques comme Goethe et Byron et les pionniers aussi hardis dans le domaine des rapports entre les sexes, tels que George Sand, ont cherché à résoudre dans la pratique de la vie ce problème compliqué, cette «énigme de l'amour ». L'auteur du roman A qui la faute?, Herzen, s'en est rendu compte à la lumière de sa propre expérience, ainsi que de nombreux autres penseurs, poètes, hommes d'État... Mais sous le poids de "l'énigme de la dualité dans l'amour » fléchissent maintenant des hommes qui ne sont pas " grands » du tout, mais qui cherchent vainement la clef de sa solution dans les limites de la pensée bourgeoise. Et cependant, cette clef est entre les mains du prolétariat. La solution de ce problème appartient à l'idéologie et au genre de vie de la nouvelle humanité laborieuse.

14 Nous parlons, ici, de la dualité du sentiment d'amour, des complexités de "l'Éros aux ailes déployées", mais une telle dualité ne peut être confondue avec les relations sexuelles d'un homme avec plusieurs femmes, ou d'une femme avec plusieurs hommes. La polygamie à laquelle le sentiment ne participe pas peut entraîner des conséquences néfastes (épuisement précoce de l'organisme, augmentation des chances dans les conditions actuelles de contracter une maladie vénérienne, etc.), mais de telles liaisons ne créent pas des «drames moraux». Les "drames", les conflits ne surgissent que lorsqu'on est en présence de l'amour dans toutes ses nuances et manifestations diverses. Une femme peut aimer un homme par "l'esprit » seulement, au cas où ses pensées, ses aspirations, ses désirs s'harmonisent avec les siens; et elle peut être 
attirée vers un autre par un puissant courant d'affinité physique. A l'égard d'une femme, un homme éprouve un sentiment d'une tendresse pleine de ménagements, d'une pitié pleine de sollicitude, et dans une autre il trouve un appui, la compréhension des meilleures aspirations de "son moi». A laquelle de ces deux femmes doit-il accorder la plénitude de l'Éros? Et pourquoi doit-il s'arracher, se mutiler l'âme si la plénitude de son être ne peut être atteinte que s'il maintient ces deux liens?

Sous le régime bourgeois, un tel dédoublement de l'âme et du sentiment entrâne d'inévitables souffrances. Pendant des siècles, l'idéologie bâtie sur l'instinct de la propriété, inculquait aux hommes que le sentiment,d'amour doit avoir comme base le principe de propriété. L'idéologie bourgeoise enfonçait dans la tête des hommes que l'amour donne le droit de posséder entièrement et sans partage le cœur de l'être aimé. Un tel idéal, une telle exclusivité dans l'amour découlait naturellement de la forme établie du mariage par couples et de l'idéal bourgeois « d'amour absorbant » entre deux époux. Mais cet idéal peut-il correspondre aux intérêts de la classe ouvrière ? N'est-il pas important et désirable au contraire du point de vue de l'idéologie prolétarienne que les sensations des hommes deviennent plus riches et plus multiples? La multiplicité de l'âme ne constitue-t-elle pas justement un fait qui facilite le développement et l'éducation des liens de cœur et d'esprit par lesquels se consolidera la collectivité laborieuse ? Plus sont nombreux les fils tendus de l'âme à l'âme, du cœur au cœur, du cerveau au cerveau, plus est solide l'esprit de solidarité et plus facilement se réalise l'idéal de la classe ouvrière : la camaraderie et l'unité.

16 L'exclusivité dans l'amour de même que « l'absorption » par l'amour ne peuvent pas, du point de vue de l'idéologie prolétarienne, constituer l'idéal d'amour déterminant les rapports entre les sexes. Au contraire, le prolétariat en constatant la multiplicité de «l'Eros aux ailes déployées " ne s'effraie point de cette découverte et n'en éprouve point d'indignation morale à l'instar de l'hypocrite bourgeoisie. Au contraire, le prolétariat cherche à imprimer à ce phénomène (qui est le résultat de causes sociales compliquées) une direction qui corresponde à ses buts de classe au moment de la lutte et de l'édification de la société communiste.

par elle-même, en contradiction avec les intérêts du prolétariat ? Au contraire, elle facilite le triomphe de l'idéal d'amour dans les rapports entre les sexes qui se forment et se cristallisent déjà à l'intérieur de la classe ouvrière : l'amour-camaraderie.

L'humanité du patriarcat se représentait l'amour sous forme d'affection entre les parents (l'amour des sœurs et des frères, l'amour envers les parents). Le monde antique mettait au-dessus de tout l'amour-amitié. Le monde féodal faisait un idéal de l'amour «spirituel» du chevalier, amour détaché du mariage et qui n'était pas lié à la satisfaction de la chair. L'idéal d'amour pour la morale bourgeoise était l'amour d'un couple uni par le mariage légitime.

19 L'idéal d'amour de la classe ouvrière découle de la collaboration dans le travail, et de la solidarité dans l'esprit et la volonté de tous ses membres hommes et femmes, il se distingue naturellement par sa forme et par son contenu de la notion d'amour d'autres époques de civilisation. Mais qu'est-ce donc que "l'amour-camaraderie »? Cela ne signifie-t-il pas que la sévère idéologie de la classe ouvrière, forgée dans une atmosphère de lutte pour la dictature ouvrière s'apprête à chasser impitoyablement le tendre Éros ailé ? Non pas. L'idéologie de la classe ouvrière non seulement ne supprime 
pas «l'Éros aux ailes déployées » mais au contraire, elle prépare la reconnaissance du sentiment d'amour en tant que force sociale et psychique.

L'hypocrite morale de la culture bourgeoise arrachait impitoyablement des plumes aux ailes à couleurs chatoyantes de l'Éros en l'obligeant à ne visiter que le «couple légalement marié ». En dehors du mariage, il n'y avait pour l'idéologie bourgeoise que l'Éros sans ailes, l'Éros déplumé - l'attraction passagère des sexes sous forme des caresses achetées (la prostitution) ou volées (l'adultère).

21 Au contraire, la morale de la classe ouvrière rejette nettement la forme extérieure qui préside aux relations d'amour entre les sexes. Pour les tâches de classe du prolétariat il est complètement indifférent que l'amour prenne la forme d'une union durable ou qu'il trouve son expression sous forme de liaison passagère. L'idéologie de la classe ouvrière ne fixe point de limites formelles à l'amour. Mais par contre elle se soucie déjà du contenu de l'amour, des nuances de sentiments et d'émotions qui lient les deux sexes. Dans ce sens l'idéologie de la classe ouvrière poursuivra bien plus impitoyablement "l'Éros sans ailes" (luxure, satisfaction unilatérale de la chair au moyen de la prostitution, transformation de l' « acte sexuel » en un but en soi, ce qui le range parmi les "plaisirs faciles ", etc.) que ne le faisait la morale bourgeoise. "L'Éros sans ailes " est en contradiction avec les intérêts de la classe ouvrière. En premier lieu il entraîne inévitablement les excès et l'épuisement physique, ce qui diminue la réserve d'énergie de l'humanité. En deuxième lieu il appauvrit l'âme en empêchant le développement des liens psychiques et des sensations sympathiques. En troisième lieu il repose sur l'inégalité des droits dans les rapports des sexes, sur la dépendance de la femme envers l'homme, sur la fatuité ou l'insensibilité de l'homme, ce qui nuit au sentiment de camaraderie. L'action de « l'Eros aux ailes déployées » est tout à fait différente.

Évidemment à la base de "l'Eros aux ailes déployées ", de même qu'à celle de l'Eros sans ailes se trouve l'attraction physique des sexes, mais la différence c'est que dans un être éprouvant un sentiment d'amour à l'égard d'un autre, s'éveillent et se manifestent justement les qualités d'âme dont les constructeurs de la nouvelle culture ont besoin: sensibilité, délicatesse, désir d'aider autrui. L'idéologie bourgeoise exigeait que l'homme ou la femme fasse montre de ces qualités rien qu'à l'égard de l'élu ou l'élue, à l'égard d'une seule femme ou d'un seul homme. Ce qui est surtout cher, par contre, à l'idéologie prolétarienne, c'est que ces qualités-là soient éveillées et éduquées dans l'homme et qu'elles se manifestent non seulement dans ses rapports avec l'objet aimé mais aussi dans ses rapports avec tous les membres de la collectivité.

Les nuances et les sentiments qui prédominent dans «l'Eros aux ailes déployées » sont indifférents au prolétariat : que ce soient les tendres tons de la complexion amoureuse ou les chaudes couleurs de la passion, ou la communauté et l'harmonie d'esprit. Une seule chose importe: c'est que dans toutes ces nuances et manifestations d'amour il existe des éléments psychiques qui développent le sentiment de camaraderie.

La reconnaissance des droits réciproques et l'art de respecter l'individualité d'un autre, même dans l'amour, le ferme appui mutuel et le souci d'aspirations collectives, tel est l'idéal de l'amour-camaraderie que se forge l'idéologie prolétarienne à la place de l'idéal d'amour conjugal « absorbant » et "exclusif » de la morale bourgeoise.

L'amour-camaraderie, c'est l'idéal dont le prolétariat a besoin dans la période difficile et grosse de responsabilité où il lutte pour instituer sa dictature ou pour la maintenir. Mais on ne peut douter que dans la société communiste une fois réalisée, l'amour, «l'Eros aux ailes déployées », se présentera sous une tout autre forme, revêtira un 
aspect tout différent de celui qu'il a aujourd'hui, un aspect complètement inconnu de nous. Les "liens sympathiques" entre les membres de la nouvelle société se développeront et se fortifieront entre temps, la «capacité d'aimer» grandira et l'amour-solidarité deviendra un animateur, comme la concurrence et l'égoïsme l'étaient pour le régime bourgeois. Le collectivisme d'esprit et de volonté vaincra l'individualisme se suffisant à lui-même. Le "froid de la solitude morale ", que les hommes cherchaient souvent à éviter en régime bourgeois, dans l'amour et le mariage, disparaîtra : les hommes seront liés entre eux par d'innombrables fils, tant de cœur que d'esprit. Les sentiments des hommes se modifieront pour faire place à l'intérêt grandissant envers la chose publique. L'inégalité entre les sexes et toutes les dépendances de la femme envers l'homme disparaîtront sans laisser de traces, complètement ensevelies dans l'oubli.

Dans cette société nouvelle, collectiviste par son esprit et ses émotions et que caractériseront l'union joyeuse et les relations fraternelles entre les membres de la collectivité laborieuse et créatrice, l'Éros prendra une place honorable en tant que sentiment multipliant la joie humaine. Quel sera cet Éros transfiguré ? La fantaisie la plus hardie est impuissante à en saisir l'aspect. Ceci seul est indiscutable: plus fortement sera soudée la nouvelle humanité par des liens durables de solidarité, plus elle sera intimement unie dans tous les domaines de la vie, de la création, et des rapports mutuels, et moins il restera de place pour l'amour dans le sens contemporain du mot. L'amour contemporain pèche toujours par ce fait qu'il absorbe toutes les pensées et tous les sentiments des "cœurs aimants» et isole et détache de la collectivité le couple aimant. Un tel détachement du " couple aimant », un tel isolement moral deviendra non seulement inutile, mais psychologiquement irréalisable, dans une société où les intérêts, les tâches, les aspirations de tous les membres seront intimement liés. Dans ce monde nouveau, la forme reconnue, normale et désirable des rapports entre les sexes aura probablement pour base la saine, la libre, la naturelle attraction des sexes (sans perversions et sans excès); elle aura pour base "l'Éros transfiguré ».

Mais nous sommes au tournant où se rencontrent les deux civilisations. Et dans cette période transitoire où les deux mondes s'empoignent chaudement sur tous les fronts, $y$ compris le front idéologique, le prolétariat est intéressé à faciliter par tous les moyens l'accumulation la plus rapide des «sensations sympathiques». Dans cette période, l'idéal moral déterminant les relations sexuelles n'est point le brutal instinct sexuel, mais les multiples sensations éprouvées aussi bien par la femme que par l'homme, d'amour-camaraderie. Pour correspondre à la nouvelle morale prolétarienne qui se forme, ces sensations doivent reposer sur les trois postulats suivants :

1. Egalité des rapports mutuels (sans la suffisance masculine et sans la dissolution servile de son individualité dans l'amour de la part de la femme);

2. Reconnaissance par l'un des droits de l'autre et réciproquement, sans prétendre posséder sans partage le cœur et l'âme de l'être aimé (sentiment de propriété, nourri par la civilisation bourgeoise);

3. Sensibilité fraternelle, art de saisir et de comprendre le travail psychique de l'être aimé (la civilisation bourgeoise n'exigeait cette sensibilité dans l'amour que chez la femme).

Mais, tout en proclamant les droits de "l'Eros aux ailes déployées» (de l'amour), l'idéologie de la classe ouvrière subordonne en même temps l'amour des membres de la collectivité laborieuse, les uns envers les autres, à un sentiment plus puissant, à un 
sentiment de devoir envers la collectivité. Quelque grand que soit l'amour unissant deux individus de sexe différent, quelque nombreux que soient les liens de cœur et d'esprit existent entre eux, les mêmes liens avec la collectivité doivent être plus forts et plus nombreux et pour ainsi dire plus organiques. La morale bourgeoise disait: tout pour l'homme aimé. La morale prolétarienne prescrit : tout pour la collectivité.

Mais j'entends déjà votre question, mon jeune ami : Soit, dites-vous, que les relations d'amour sur la base de l'esprit fraternel deviennent l'idéal de la classe ouvrière, mais cet idéal, cette nouvelle " mesure morale » de l'amour, ne pèsera-t-elle pas lourdement sur les sentiments d'amour? Ne chiffonnera-t-elle pas, ne mutilera-t-elle pas les tendres ailes de "l'ombrageux Eros"? Ayant libéré l'amour des chaînes de la morale bourgeoise, ne lui en créons-nous pas de nouvelles?

Oui, mon jeune ami, vous avez raison. L'idéologie du prolétariat, tout en rejetant la "morale» bourgeoise dans le domaine des relations matrimoniales, se forge inévitablement sa propre morale de classe, ses nouvelles règles des rapports entre les sexes, qui correspondent mieux aux tâches de la classe ouvrière, qui éduquent les sentiments de ses membres et qui par là constituent, jusqu'à un certain point, des chaînes pour le sentiment. Dans la mesure où il s'agit d'amour patronné par l'idéologie bourgeoise, le prolétariat arrachera incontestablement un grand nombre de plumes aux ailes de l'Éros, tel que cette idéologie se le représente. Mais regretter que la classe laborieuse marque également de son sceau les rapports entre les sexes afin de faire correspondre le sentiment d'amour à la tâche qui est la sienne, ce n'est pas regarder l'avenir. Il est évident qu'à la place d'anciennes plumes arrachées aux ailes de l'Éros, la classe ascendante en fera croître d'autres, d'une beauté, d'une puissance et d'un éclat encore inconnus. N'oubliez pas, mon jeune ami, que l'amour change inévitablement d'aspect et se transforme avec la base économique et culturelle de l'humanité.

Si dans les rapports d'amour s'atténue l'aveugle, l'exigeant, l'absorbant sentiment passionnel, si celui de propriété y disparait, ainsi que le désir égoïste de s'attacher "pour toujours» l'être aimé, s'il y disparaît enfin la fatuité de l'homme et la renonciation criminelle à son «moi» de la part de la femme, par contre, d'autres éléments précieux de l'amour se développeront. Le respect de la personnalité d'autrui grandira, l'art de compter avec les droits des autres se perfectionnera, la sensibilité réciproque grandira et se développera en même temps la tendance à manifester l'amour non seulement en baisers et embrassades, mais aussi dans l'unité d'action et de volonté dans la création commune.

\section{INDEX}

Mots-clés : Amitié érotique, libération sexuelle, Eros, révolution russe, lutte des classes 
AUTEUR

ALEXANDRA KOLLONTAI

Walter Benjamin fut une figure centrale de la révolution russe et de l'opposition de gauche en Union soviétique 